\title{
Fragmented Sleep and the Prevalence of Hypertension in Middle-Aged and Older Individuals
}

\author{
Juan Zhao' \\ Wenjuan Wang' \\ Suhua Wei ${ }^{1}$ \\ Lihong Yang ${ }^{2}$ \\ Yanhua $\mathrm{Wu}^{2}$ \\ Bin Yan (ID) ${ }^{2,3}$
}

'Department of Haematology, the First Affiliated Hospital of Xi'an Jiaotong University, Xi'an, People's Republic of China; ${ }^{2}$ Department of Clinical Research Center, the First Affiliated Hospital of Xi'an Jiaotong University, Xi'an, People's Republic of China; ${ }^{3}$ Department of Psychiatry, the First Affiliated Hospital of Xi'an Jiaotong University, Xi'an, People's Republic of China
Correspondence: Bin Yan Department of Clinical Research Center, the First Affiliated Hospital of Xi'an Jiaotong University, Xi'an, People's Republic of China

Tel +8602985323614

Email yanbin.1987@hotmail.com
Objective: We aimed to investigate the association between fragmented sleep and the prevalence of hypertension in middle-aged and older individuals.

Methods: This study included 5804 participants with an average age of $63.1 \pm 11.2$ years from the Sleep Heart Health Study. Fragmented sleep parameters including arousal index in total sleep (ArI-Total), rapid eye movement sleep (ArI-REM), non-rapid eye movement sleep (ArI-NREM), fragmented sleep index (SFI), sleep efficiency (SE) and wake after sleep onset (WASO) were monitored using polysomnography. The information on hypertension, defined as systolic blood pressure (SBP) $\geq 140 \mathrm{mmHg}$ and/or diastolic blood pressure (DBP) $\geq 90$ mmHg or under antihypertensive treatment, was collected at baseline. We conducted multivariable logistic regression to explore the cross-sectional association between fragmented sleep and the prevalence of hypertension.

Results: After adjusting for potential confounders, fragmented sleep parameters (per 5-unit change) including SE (odds ratio [OR] 0.904; 95\% confidence interval [CI] 0.877-0.932; P < 0.001), WASO (OR 1.019; 95\% CI 1.012-1.027; P < 0.001), ArI-Total (OR, 1.036; 95\% CI, $1.005-$ $1.068 ; \mathrm{P}=0.024$ ), and ArI-NREM (OR 1.032; 95\% CI 1.004-1.062; $\mathrm{P}=0.027$ ) were significantly associated with the prevalence of hypertension. In addition, ArI-Total, ArI-NREM, and ArI-REM were positively correlated with both systolic blood pressure and diastolic blood pressure.

Conclusion: We found a high prevalence of hypertension among middle-aged and older individuals with fragmented sleep. The causal association between fragmented sleep and hypertension warrants further investigation.

Keywords: blood pressure, polysomnography, fragmented sleep, Sleep Heart Health Study, cross-sectional study

\section{Introduction}

Hypertension, also known as elevated blood pressure, is a global public health issue. Approximately 1.28 billion people age 30-79 years have hypertension worldwide, and the number of people with systolic blood pressure (SBP) of $140 \mathrm{mmHg}$ or higher increased from 17,307 to 20,526 per 100,000 between 1990 and $2015 .^{1,2}$ Individuals with hypertension have an increased risk of heart, brain, and kidney disease. $^{3}$ Hypertension is the most common preventable risk factor for cardiovascular disease; the prevention of hypertension would decrease the social burden associated with the condition and improve the quality of life. ${ }^{4-6}$

Previous studies have found a close relationship between sleep and hypertension. ${ }^{7,8}$ Individuals with poor sleep quality have higher average SBP and 
diastolic blood pressure (DBP). ${ }^{9}$ Short sleep duration is a risk factor for hypertension, ${ }^{10}$ and sleep-disordered breathing may lead to an increased risk of the condition. ${ }^{11}$ Fragmented sleep is another sleep condition that can be monitored using wrist actigraphy. ${ }^{12}$ A previous study based on wrist actigraphy reported that individuals with low sleep efficiency (SE) had high daytime SBP but not DBP. ${ }^{13}$

Polysomnography (PSG) can record more fragmented sleep parameters than wrist actigraphy, including arousal index (ArI) in total sleep (ArI-Total), sleep fragmentation index (SFI), SE, and wake after sleep onset (WASO). Furthermore, PSG can be used to monitor the ArI in rapid eye movement sleep (ArI-REM) and non-rapid eye movement sleep (ArI-NREM). There is little evidence regarding the association between fragmented sleep and hypertension in NREM and REM sleep based on PSG records. In this study, we aimed to explore the relationship between fragmented sleep and the prevalence of hypertension using data from the Sleep Heart Health Study (SHHS).

\section{Materials and Methods Study Population}

This study included individuals from community-based populations examined in the SHHS study. The SHHS (ClinicalTrials.gov Identifier: NCT00005275) recruited participants who met the inclusion criteria (age 40 years or older; no history of treatment of sleep apnea; no tracheostomy; no current home oxygen therapy) from nine existing epidemiological studies, including the Framingham Offspring Cohort, the Hagerstown and Minneapolis/St. Paul sites of the Atherosclerosis Risk in Communities (ARIC) study, the Hagerstown, Sacramento and Pittsburgh sites of the Cardiovascular Health Study (CHS), the Strong Heart Study sites in South Dakota, Oklahoma and Arizona, and Studies of respiratory disease in Tucson and of hypertension in New York. But the Strong Heart Study participants were excluded due to sovereignty issues. Several parent cohorts oversampled snorers to increase the study-wide prevalence of sleepdisordered breathing. There was a clear and rigorous Data Quality Assurance and Control system in each parent study. For the existing data collected by the parent studies, the Comparability Committee was charged with comparing data collected by the various parent studies to determine the data to be used. All participants provided written informed consent, and the study was approved by the institutional review board of each institution. The study design and quality control procedures were previously reported. ${ }^{14}$ We also provided details about the PSG monitor in Supplement Methods. We had access to the SHHS database through a signed agreement with the Brigham and Women's Hospital. This research was approved by the Ethical Review Board of the First Affiliated Hospital of Xi'an Jiaotong University.

\section{Fragmented Sleep Parameters}

All the participants underwent overnight unattended PSG at home using a portable monitor (P-Series; Compumedics, Abbotsville, Australia) between November 1995 and January 1998. ArI-total was calculated as the total number of sleep arousals divided by the total sleep time. ArI-NREM and ArI-REM were measured based on the number of arousals per hour during NREM and REM sleep, respectively. SFI was defined as the number of awakenings and sleep-stage shifts per hour. SE was calculated as the total sleep time divided by the total time in bed and multiplied by $100 \%$. WASO was defined as the total awake time from the first time the participant fell asleep to the time the participant was fully awake. Fragmented sleep parameters were also divided into four groups based on quartiles to observe the prevalence of hypertension in different group.

\section{Outcomes and Covariates}

All of the initial blood pressure measurements were performed in the subject's home, prior to setting up the PSG equipment. Blood Pressure was taken by the sleep technician with the subject in a seated position after 5 minutes' rest at baseline of SHHS, using a standard mercury sphygmomanometer and a choice of four cuff sizes. The observer listened with the bell of a Littman stethoscope and recorded the pressures. The average of the two readings taken with at least 30 second intervals was used to describe the blood pressure. Hypertension status was based on blood pressure measurements (SBP $\geq 140$ $\mathrm{mmHg}$ and/or DBP $\geq 90 \mathrm{mmHg}$ ) or antihypertensive treatment (mainly included angiotensin-converting enzyme inhibitors, Beta-blockers, calcium-channel blocker, diuretic and vasodilators). Covariates including age, gender, race, smoking status, alcohol use, body mass index (BMI), benzodiazepine use and history of diabetes mellitus were collected from the SHHS baseline investigations. Sleep duration was based on the total sleep time according 
to the PSG records. The apnea-hypopnea index (AHI) was defined as the number of apnea and hypopnea episodes (at least $4 \%$ decrease in oxygen saturation) divided by the total sleep time. In the present study, sleep disordered breathing (SDB) was defined as an AHI of $\geq 5$ events/h.

\section{Statistical Analyses}

Differences in baseline characteristics between hypertensive and non-hypertensive patients were assessed using chi-square tests for categorical variables and independentsample $t$-tests for continuous variables. Univariable and multivariable logistic regression analyses were employed to explore the cross-sectional associations between fragmented sleep parameters and the prevalence of hypertension. We also investigated the correlations between fragmented sleep parameters and SBP/DBP using linear regression analysis. Multicollinearity among the different fragmented sleep parameters was examined using multivariable regression analysis. Interaction analysis stratified by SDB (AHI $<5$ events/h vs AHI $\geq 5$ events/h), age ( $\geq 60$ vs $<60$ ), gender (males vs females) and alcohol use (yes vs no) were also conducted. All analyses were performed using SPSS software (v.24.0, SPSS Inc., Chicago, IL). Two-sided P-values $<0.05$ indicated statistical significance.

\section{Results}

\section{Participant Characteristics}

A total of 5804 middle-aged and older adults (2765 men and 3039 women with an average age of $63.1 \pm 11.2$ years) were included in our study. Characteristics of the hypertensive and non-hypertensive participants are shown in Table 1. Hypertension was more prevalent among participants who were older, with a high BMI, and with diabetes mellitus. In addition, when compared to controls, participants with hypertension had significantly elevated ArI-Total, ArIREM, ArI-NREM, SFI, WASO, and AHI and reduced SE.

\section{Fragmented Sleep Parameters and Hypertension}

The distributions of hypertension differed significantly in the different fragmented sleep quartiles (Figure 1). Participants with elevated ArI-Total $(\mathrm{P}<0.001)$, ArINREM $(\mathrm{P}<0.001)$ and WASO $(\mathrm{P}<0.001)$ tended to have an increased prevalence of hypertension; individuals with high SE had a lower prevalence of hypertension than those with low SE $(\mathrm{P}<0.001)$.
Univariable and multivariable logistic regression analyses were further used to investigate the relationships between fragmented sleep parameters and hypertension. Multicollinearity was found among the different fragmented sleep parameters. Therefore, we explored the role of each individual fragmented sleep variable on hypertension risk after adjusting for age, gender, race, BMI, smoking status, alcohol use, diabetes mellitus, sleep duration, benzodiazepine use, triglyceride level, total cholesterol, and AHI. Our results (per 5-unit increased) showed that SE (odds ratio [OR] 0.904; confidence interval $[\mathrm{CI}]$ 0.877-0.932; $\mathrm{P}<0.001)$, WASO (OR 1.019; 95\% CI 1.012-1.027; $\mathrm{P}<0.001$ ), ArI-Total (OR, 1.036; 95\% CI, 1.005-1.068; $\mathrm{P}=0.024$ ), and ArINREM (OR 1.032; 95\% CI 1.004-1.062; $\mathrm{P}=0.027$ ) were significantly associated with the prevalence of hypertension (Table 2). No significant association of SFI and ArI-REM with the prevalence of hypertension was found.

\section{Fragmented Sleep Parameters and SBP/ DBP}

The correlations between each individual fragmented sleep parameter and SBP/DBP were also determined using multivariable linear regression analysis. ArI-Total $(\beta$ 0.094; $95 \%$ CI 0.041-0.146; $\mathrm{P}<0.001)$, ArI-NREM ( $\beta$ 0.080; 95\% CI $0.032-0.128 ; \mathrm{P}=0.001)$, ArI-REM $(\beta 0.061 ; 95 \%$ CI 0.010 0.112; $\mathrm{P}=0.018$ ), and WASO ( $\beta$ 0.014; 95\% CI 0.002-0.026; $\mathrm{P}=0.022)$ were positively correlated with SBP, while SE ( $\beta$ $-0.126 ; 95 \% \mathrm{CI}-0.178$ to $-0.075 ; \mathrm{P}<0.001)$ was negatively associated with SBP. In addition, ArI-Total $(\beta 0.033 ; 95 \% \mathrm{CI}$ $0.002-0.064 ; \mathrm{P}=0.034)$, ArI-NREM ( $\beta 0.029 ;$ 95\% CI 0.001$0.057 ; \mathrm{P}=0.041)$ and ArI-REM $(\beta \quad 0.047 ; 95 \%$ CI 0.018 $0.077 ; \mathrm{P}=0.002$ ) were significantly correlated with $\mathrm{DBP}$ (Table 3).

\section{Interaction Analysis}

Individuals with SDB may experience micro-arousal. Therefore, we investigated the association between fragmented sleep parameters and hypertension stratified by SDB (AHI $\geq 5$ events/h vs AHI $<5$ events/h). We further explored the relationship between fragmented sleep parameters and hypertension stratified by age ( $\geq 60$ vs $<60$ ), gender (males vs females) and alcohol use (yes vs no). No significant interaction was found in these analyses $\left(\mathrm{P}_{\text {interaction }}>0.05\right)(\underline{\text { Supplement Tables 1- }}-\underline{4})$. 
Table I Subject Characteristics in Participants with or Without Hypertension

\begin{tabular}{|c|c|c|c|c|}
\hline Characteristics & Total $(n=5804)$ & Hypertension $(n=2948)$ & Controls $(n=2856)$ & $P$ value \\
\hline Age, years & $63.1 \pm 11.2$ & $66.4 \pm 10.6$ & $59.8 \pm 10.8$ & $<0.001$ \\
\hline Gender, n (\%) & & & & 0.061 \\
\hline Male & 2765 (47.7) & 1440 (48.8) & $1325(46.4)$ & - \\
\hline Female & $3039(52.3)$ & $1508(5 \mid .2)$ & I53| (53.6) & - \\
\hline Race, n (\%) & & & & $<0.001$ \\
\hline White & $4907(84.6)$ & $2483(84.3)$ & $2424(84.9)$ & - \\
\hline Black & $515(8.9)$ & 327 (II.I) & $188(6.6)$ & - \\
\hline Other & $380(6.5)$ & $137(4.6)$ & $243(8.5)$ & - \\
\hline BMI, kg/m² & $28.2 \pm 5.1$ & $28.8 \pm 5.4$ & $27.5 \pm 4.7$ & $<0.001$ \\
\hline Smoking status, n (\%) & & & & $<0.001$ \\
\hline Current smoker & $560(9.7)$ & $224(7.5)$ & $336(11.4)$ & - \\
\hline Former smoker & $2495(43.3)$ & I305 (45.0) & II $90(42.0)$ & - \\
\hline Never smoker & $2708(47.0)$ & I 399 (47.5) & I309 (46.6) & - \\
\hline Alcohol use, n (\%) & & & & $<0.001$ \\
\hline At least I drink per day & $2412(44.8)$ & 1160 (42.2) & $1252(47.4)$ & \\
\hline None & 2977 (55.2) & I590 (57.8) & I387 (52.6) & \\
\hline Benzodiazepine use, n (\%) & $308(5.3)$ & $201(6.8)$ & $107(3.9)$ & $<0.001$ \\
\hline Diabetes mellitus, n (\%) & $405(7.0)$ & $311(10.5)$ & $94(3.3)$ & $<0.001$ \\
\hline Sleep duration, n (\%) & & & & $<0.001$ \\
\hline$<6$ hours & $624(10.8)$ & $366(12.4)$ & $258(9.1)$ & - \\
\hline $6-8$ hours & 3784 (65.2) & $|88|(63.8)$ & $1903(66.6)$ & - \\
\hline$>8$ hours & $1396(24.0)$ & $70 \mathrm{I}(23.8)$ & $695(24.3)$ & - \\
\hline \multicolumn{5}{|l|}{ Sleep fragmentation } \\
\hline SE, \% & $82.8 \pm 10.6$ & $81.0 \pm 11.2$ & $84.6 \pm 9.5$ & $<0.001$ \\
\hline WASO, min & $61.4 \pm 44.0$ & $67.4 \pm 46.5$ & $55.3 \pm 40.4$ & $<0.001$ \\
\hline SFI, events/h & $9.0 \pm 3.5$ & $9.2 \pm 3.8$ & $8.8 \pm 3.2$ & $<0.001$ \\
\hline Arl-Total, events/h & $19.2 \pm 10.7$ & $20.2 \pm 11.7$ & $18.1 \pm 9.4$ & $<0.001$ \\
\hline Arl-REM, events/h & $15.5 \pm 10.9$ & $15.9 \pm 11.9$ & $15.0 \pm 9.9$ & 0.002 \\
\hline Arl-NREM, events/h & $20.0 \pm 11.6$ & $21.2 \pm 12.7$ & $19.1 \pm 10.6$ & $<0.001$ \\
\hline$A H I$, events/h & $10.2 \pm 13.6$ & $12.1 \pm 15.3$ & $8.2 \pm 11.3$ & $<0.001$ \\
\hline SBP, mmHg & $127.4 \pm 19.3$ & $137.7 \pm 19.6$ & $116.6 \pm 11.6$ & $<0.001$ \\
\hline DBP, mmHg & $73.7 \pm 11.6$ & $76.8 \pm 13.1$ & $70.4 \pm 8.7$ & $<0.001$ \\
\hline Triglycerides, mg/dl & $150.1 \pm 101.1$ & $159.7 \pm 98.9$ & $140.0 \pm 102.3$ & $<0.001$ \\
\hline Cholesterol, mg/dl & $207.6 \pm 38.7$ & $209.4 \pm 38.7$ & $205.7 \pm 38.6$ & 0.001 \\
\hline
\end{tabular}

Notes: Results are presented as mean \pm standard deviation or $\mathrm{n}(\%)$. The $\mathrm{P}$ values represent the difference between two groups.

Abbreviations: AHI, apnea hypopnea index; Arl, arousal index; BMI, body mass index; DBP, diastolic blood pressure; NREM, non-rapid eye movement; REM, rapid eye movement; SBP, systolic blood pressure; SE, sleep efficiency; SFI, sleep fragmentation index; WASO, wake after sleep onset.

\section{Discussion}

In this large community-based study, we investigated the cross-sectional associations between fragmented sleep parameters and hypertension prevalence. Fragmented sleep variables, including ArI-Total, ArI-NREM, SE, and WASO, were significantly associated with the prevalence of hypertension. Moreover, ArI-Total, ArI-NREM and ArI-REM were positively correlated with both SBP and DBP, and increased SE was associated with a lower SBP.

Fragmented sleep usually refers to arousals over the course of the entire sleep period. Physical and mental diseases as well as lifestyle habits such as exercising before bedtime, long naps during the day, alcohol use, caffeine consumption and a poor sleep environment may lead to fragmented sleep. ${ }^{15}$ SDB accompanied by microarousal also contributes to fragmented sleep. ${ }^{16}$ Previous studies have shown that fragmented sleep promotes obesity in mice, ${ }^{17}$ and this association has also been found in humans. ${ }^{18}$ Several studies have explored the relationship between fragmented sleep and blood pressure. Morrell et al found that SFI was associated with a high level of awake SBP, but they found no significant association 
A

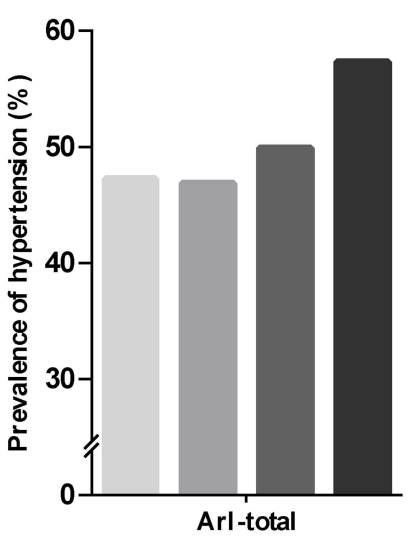

D

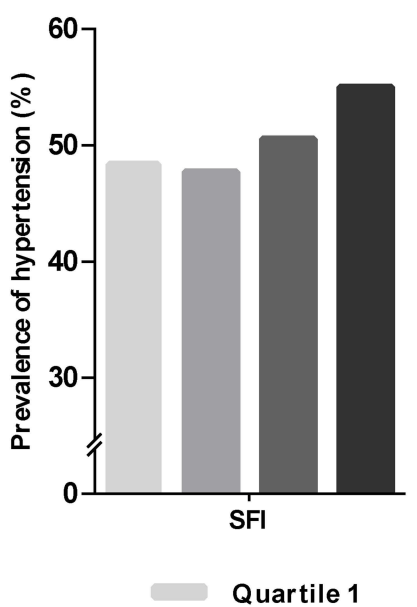

B

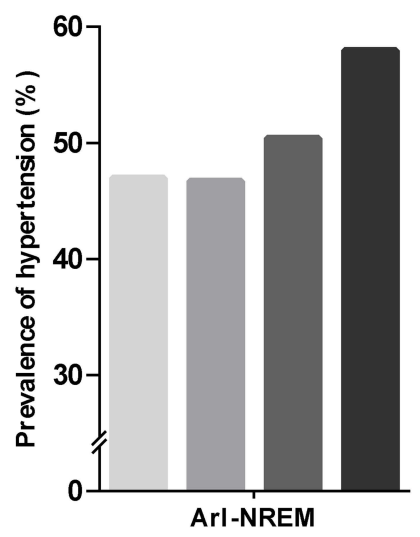

E

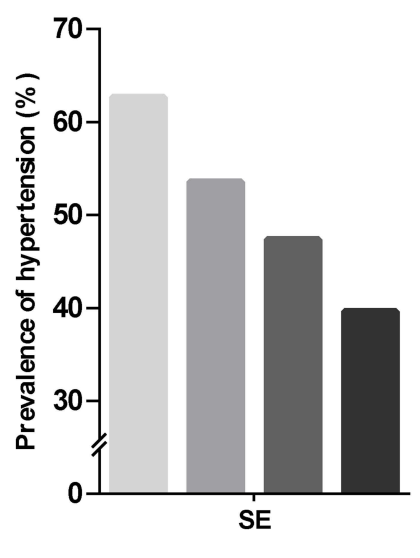

C

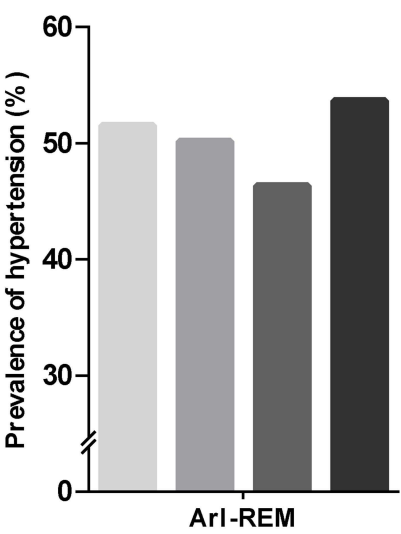

$\mathbf{F}$

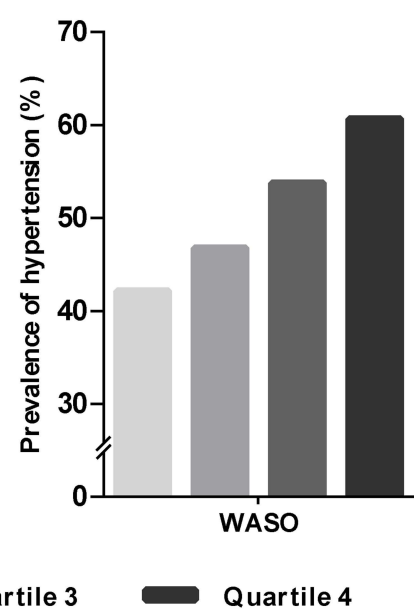

Figure I The distribution of prevalent hypertension in different fragmented sleep quartiles. (A) Arl-Total (quartile I: $<12.0$ events/h, quartile $2:$ I2.0-I6.7 events/h, quartile 3: I6.8-23.5 events/h, and quartile 4: $\geq 23.6$ events/h); (B) Arl-NREM (quartile I: $<12.1$ events/h, quartile 2: I2. I-I7.2 events/h, quartile 3: I7.3-24.8 events/h, and quartile 4: $\geq 24.9$ events/h); (C) ArI-REM (quartile I: $<7.8$ events/h, quartile 2: 7.8-12.9 events/h, quartile 3: 13.0-20.2 events/h, and quartile 4: $\geq 20.3$ events/h); (D) SFI (quartile I: $<6.7$ events/h, quartile 2: 6.7-8.5 events/h, quartile 3: 8.6-10.6 events/h, and quartile 4: $\geq 10.7$ events/h); (E) SE (quartile $1:<77.7 \%$, quartile $2: 77.7 \%-85.0 \%$, quartile 3: $85.1 \%$ 90.4\%, and quartile 4: $\geq 90.5 \%$ ); (F) WASO (quartile I: <29.6min; quartile 2: 29.6-49.4 min; quartile 3: $49.5-81.9$ min; quartile 4: $>82.0 \mathrm{~min}$ ).

between the SFI and awake SBP after adjusting for AHI. ${ }^{19}$ Doyle et al found that SE was positively associated with average daytime SBP, but not DBP, using wrist actigraphy. ${ }^{13}$ In the present study, we also investigated the correlations between fragmented sleep parameters and SBP and DBP. Our results showed that SE and WASO were associated with SBP, but not with DBP, based on PSG records. In addition, no significant associations were found between SFI and SBP and DBP. Ramos et al revealed that a $10 \%$ reduction in $\mathrm{SE}$ was associated with a $7.5 \%$ increase in hypertension prevalence based on wrist actigraphy. ${ }^{20}$ In our study, SE and WASO were significantly associated with the prevalence of hypertension. Our results also indicated that elevated ArI-Total and ArI-NREM increased the risk of hypertension. Furthermore, we found that ArI was positively correlated with both SBP and DBP in total sleep time, NREM sleep, and REM sleep. Our findings showed that individuals with fragmented sleep had a higher level of SBP/DBP and a higher prevalence of hypertension. Improving fragmented sleep may aid in controlling human blood pressure and decreasing the risk of hypertension.

The relationship between hypertension and sleep disturbances is complex. Some conditions such as age, gender, SDB and alcohol use may contribute to both sleep disorder and hypertension which may be the mediate or confounders. ${ }^{8}$ Previous studies have shown that SDB is a risk factor for hypertension. ${ }^{11,21} \mathrm{SDB}$ may be accompanied by micro-arousal, which may further lead to fragmented sleep. AHI is usually used to assess the severity of $\mathrm{SDB}^{22}$ Our multicollinearity analysis revealed that AHI had some effect on ArI. Fragmented sleep parameters, 
Table 2 ORs and 95\% Cls for Sleep Fragmentation Associated with Hypertension

\begin{tabular}{|c|c|c|c|c|c|c|}
\hline \multirow[t]{2}{*}{ Sleep Fragmentation* } & \multicolumn{2}{|c|}{ Unadjusted } & \multicolumn{2}{|c|}{ Multivariable Adjusted $^{\mathbf{a}}$} & \multicolumn{2}{|c|}{ Multivariable Adjusted ${ }^{\mathbf{b}}$} \\
\hline & OR $(95 \% \mathrm{Cl})$ & $P$ value & OR $(95 \% \mathrm{Cl})$ & $P$ value & OR $(95 \% \mathrm{Cl})$ & $P$ value \\
\hline SE & 0.845 (0.823 to 0.867$)$ & $<0.001$ & 0.901 (0.874 to 0.929$)$ & $<0.001$ & 0.904 (0.877 to 0.932$)$ & $<0.001$ \\
\hline WASO & 1.033 (I.027 to 1.039$)$ & $<0.001$ & 1.020 (I.013 to 1.028$)$ & $<0.001$ & 1.019 (1.012 to 1.027$)$ & $<0.001$ \\
\hline SFI & $1.176(1.091$ to 1.267$)$ & $<0.001$ & 1.092 (1.000 to 1.192$)$ & 0.050 & 1.078 (0.987 to 1.177$)$ & 0.096 \\
\hline Arl-Total & 1.097 (I.069 to I.I25) & $<0.001$ & 1.048 (1.017 to 1.079$)$ & 0.002 & $1.036(1.005$ to 1.068$)$ & 0.024 \\
\hline Arl-REM & $1.038(1.014$ to 1.064$)$ & 0.002 & I.03I (I.002 to I.06I) & 0.039 & 1.021 (0.99I to I.05I) & 0.174 \\
\hline Arl-NREM & $1.090(1.065$ to 1.116$)$ & $<0.001$ & $1.042(1.014$ to $1.07 \mathrm{I})$ & 0.003 & 1.032 (1.004 to 1.062$)$ & 0.027 \\
\hline
\end{tabular}

Notes: *Per 5-unit increased. The results between SE, WASO, SFI and hypertension were based on 5804 individuals. The results between Arl-Total, Arl-REM, Arl-NREM and hypertension was based on 5642 individuals. ${ }^{a}$ Each individual sleep fragmentation variables including Arl-Total, Arl-REM, Arl-NREM, SFI, SE and WASO was adjusted for age, gender, race, BMI, smoking status, alcohol use, diabetes mellitus, sleep duration, benzodiazepine use, triglyceride and total cholesterol. ${ }^{b} A d j u s t e d$ for a+ AHI.

Abbreviations: $\mathrm{AHI}$, apnea hypopnea index; Arl, arousal index; $\mathrm{BMI}$, body mass index; $95 \% \mathrm{Cl}, 95 \%$ confidence interval; NREM, non-rapid eye movement; OR, odds ratio; REM, rapid eye movement; SE, sleep efficiency; SFI, sleep fragmentation index; WASO, wake after sleep onset.

including ArI-Total, ArI-NREM, ArI-REM, SE, and WASO, remained significantly associated with the prevalence of hypertension whether we adjusted for AHI in the multivariable analysis. Interaction analysis stratified by SDB and non-SDB showed no significant interaction when exploring the associations between each fragmented sleep variable and hypertension. Furthermore, no significant interaction was found when performed the subgroup analysis stratified by age, gender and alcohol use.

The mechanisms underlying the association between fragmented sleep and hypertension are not fully understood. It is possible that fragmented sleep increases the activity of the sympathetic nervous system, which then further contributes to elevated blood pressure. $^{17}$ Fragmented sleep is also associated with decreased physical activity, insulin resistance, obesity, and increased inflammation. ${ }^{23-26}$ In addition, fragmented sleep may increase the body's stress response and lead to short sleep, poor sleep quality, and more daytime naps, all of which may contribute to high blood pressure. ${ }^{27-29}$

The present study has several strengths. Our analysis was based on a large community population from the SHHS datasets, and all fragmented sleep parameters were objectively monitored using PSG. We investigated the association between fragmented sleep and hypertension in total sleep time, NREM sleep, and REM sleep. However,

Table 3 Univariable and Multivariable Linear Regression Analysis for Sleep Fragmentation Associated with SBP and DBP

\begin{tabular}{|c|c|c|c|c|c|c|}
\hline \multirow[t]{2}{*}{ Variables } & \multicolumn{2}{|l|}{ Unadjusted } & \multicolumn{2}{|c|}{ Multivariable Adjusted $^{\mathrm{a}}$} & \multicolumn{2}{|c|}{ Multivariable Adjusted $^{\mathbf{b}}$} \\
\hline & $\beta(95 \% \mathrm{Cl})$ & $\mathbf{P}$ value & $\beta(95 \% \mathrm{Cl})$ & $P$ value & $\beta(95 \% \mathrm{Cl})$ & P value \\
\hline \multicolumn{7}{|l|}{ SBP } \\
\hline SE & $-0.243(-0.290$ to -0.196$)$ & $<0.001$ & $-0.131(-0.182$ to -0.080$)$ & $<0.001$ & $-0.126(-0.178$ to -0.075$)$ & $<0.001$ \\
\hline WASO & 0.04 I (0.029 to 0.052$)$ & $<0.001$ & 0.015 (0.003 to 0.028$)$ & 0.014 & $0.014(0.002$ to 0.026$)$ & 0.022 \\
\hline SFI & $0.103(-0.039$ to 0.245$)$ & 0.155 & $-0.058(-0.209$ to 0.093$)$ & 0.449 & $-0.079(-0.230$ to 0.073$)$ & 0.307 \\
\hline Arl-Total & 0.181 (0.134 to 0.229$)$ & $<0.001$ & 0.107 (0.056 to 0.158$)$ & $<0.001$ & $0.094(0.04 \mathrm{I}$ to 0.146$)$ & $<0.001$ \\
\hline Arl-REM & $0.090(0.043$ to 0.136$)$ & $<0.001$ & 0.074 (0.025 to 0.124$)$ & 0.003 & 0.061 (0.010 to 0.112$)$ & 0.018 \\
\hline Arl-NREM & $0.164(0.121$ to 0.208$)$ & $<0.001$ & 0.092 (0.045 to 0.139$)$ & $<0.001$ & 0.080 (0.032 to 0.128$)$ & 0.001 \\
\hline \multicolumn{7}{|l|}{ DBP } \\
\hline SE & $0.013(-0.016$ to 0.041$)$ & 0.392 & $-0.015(-0.045$ to 0.014$)$ & 0.312 & $-0.014(-0.044$ to 0.016$)$ & 0.368 \\
\hline WASO & $-0.013(-0.020$ to -0.006$)$ & $<0.001$ & $-0.003(-0.010$ to 0.004$)$ & 0.444 & $-0.003(-0.010$ to 0.004$)$ & 0.379 \\
\hline SFI & $-0.070(-0.155$ to 0.015$)$ & 0.106 & $-0.054(-0.141$ to 0.034$)$ & 0.231 & -0.061 ( -0.149 to 0.027$)$ & 0.174 \\
\hline Arl-Total & 0.059 (0.03। to 0.088$)$ & $<0.001$ & $0.039(0.009$ to 0.069$)$ & 0.010 & 0.033 (0.002 to 0.064$)$ & 0.034 \\
\hline Arl-REM & $0.099(0.07 \mid$ to 0.127$)$ & $<0.001$ & $0.052(0.023$ to $0.08 \mathrm{I})$ & $<0.001$ & 0.047 (0.018 to 0.077$)$ & 0.002 \\
\hline Arl-NREM & $0.04 \mathrm{I}(0.014$ to 0.067$)$ & 0.003 & 0.030 (0.003 to 0.058$)$ & 0.031 & $0.029(0.001$ to 0.057$)$ & 0.041 \\
\hline
\end{tabular}

Notes: The results between SE, WASO, SFI and SBP/DBP were based on 569I individuals. The results between Arl-Total, Arl-REM, ArI-NREM and SBP/DBP was based on 5536 individuals. ${ }^{a}$ Each individual sleep fragmentation variables including Arl-Total, Arl-REM, Arl-NREM, SFI, SE and WASO was adjusted for age, gender, race, BMI, smoking status, alcohol use, diabetes mellitus, sleep duration, benzodiazepine use, triglyceride and total cholesterol. ${ }^{\mathrm{b}} \mathrm{Adjusted}$ for a+ AHI.

Abbreviations: $\mathrm{AHI}$, apnea hypopnea index; Arl, arousal index; $\mathrm{BMI}$, body mass index; $95 \% \mathrm{Cl}, 95 \%$ confidence interval; DBP, diastolic blood pressure; NREM, non-rapid eye movement; REM, rapid eye movement; SBP, systolic blood pressure; SE, sleep efficiency; SFI, sleep fragmentation index; WASO, wake after sleep onset. 
there are several potential limitations to the study that should be noted. First, we found a cross-sectional association between fragmented sleep and hypertension, but the causal relationship requires further study. Second, a singlenight PSG may not fully represent an individual's sleep characteristics. Wrist actigraphy has an advantage as it allows for multiple sleep monitoring. Thirdly, the definition of hypertension in our study was based on the blood pressure measurement and information about antihypertensive treatment. It is difficult to identify the masked hypertension in our study. Ambulatory blood pressure monitoring may be a useful measurement method. ${ }^{30}$ In SHHS, scorers reviewed the record, on an epoch by epoch basis (on screen), marking each sleep stage, each arousal, and each respiratory event. All the sleep technicians and investigators were trained and the portable monitor were corrected in SHHS study, but the information bias could not be completely avoided. Finally, as our findings were based on middle-aged and older individuals, they may not extrapolate to the entire population.

\section{Conclusion}

In conclusion, our study provides evidence that middleaged and older individuals with fragmented sleep have an increased risk of hypertension. Elevated ArI-Total, ArINREM, and ArI-REM were positively correlated with high SBP and DBP. Our results indicate that ArI-Total, ArI-NREM, SE, and WASO were significantly associated with the prevalence of hypertension. Improving fragmented sleep may help control high blood pressure. The causal relationship between fragmented sleep and hypertension also deserves further investigation.

\section{Author Responsibility Information}

All authors made a significant contribution to the work reported, whether that is in the conception, study design, execution, acquisition of data, analysis and interpretation, or in all these areas; took part in drafting, revising or critically reviewing the article; gave final approval of the version to be published; have agreed on the journal to which the article has been submitted; and agree to be accountable for all aspects of the work.

\section{Acknowledgments}

We appreciate the Brigham and Women's Hospital for sharing the Datasets of Sleep Heart Health Study (SHHS). The Sleep Heart Health Study (SHHS) was supported by National Heart, Lung, and Blood Institute cooperative agreements
U01HL53916 (University of California, Davis), U01HL53931 (New York University), U01HL53934 (University of Minnesota), U01HL53937 and U01HL64360 (Johns Hopkins University), U01HL53938 (University of Arizona), U01HL53940 (University of Washington), U01HL53941 (Boston University), and U01HL63463 (Case Western Reserve University). The National Sleep Research Resource was supported by the National Heart, Lung, and Blood Institute (R24 HL114473, 75N92019R002). SHHS is particularly grateful to the members of these cohorts who agreed to participate in SHHS as well. SHHS further recognizes all the investigators and staff who have contributed to its success. A list of SHHS investigators, staff and their participating institutions is available on the SHHS website, https://doi. org/10.25822/ghy8-ks59.

\section{Funding}

This research was supported by Key Research and Development Program of Shaanxi (No. 2020SF-169) and Natural Science Basic Research Program of Shaanxi (No. 2021JQ-395).

\section{Disclosure}

All the authors declare no conflicts of interest in this work.

\section{References}

1. Forouzanfar MH, Liu P, Roth GA, et al. Global burden of hypertension and systolic blood pressure of at least 110 to $115 \mathrm{~mm} \mathrm{Hg}, 1990$ 2015. JAMA. 2017;317:165-182. doi:10.1001/jama.2016.19043

2. WHO. Hypertension; 2021. Available from: https://wwwwhoint/ news-room/fact-sheets/detail/hypertension. Accessed December 24, 2021.

3. Oparil S, Acelajado MC, Bakris GL, et al. Hypertension. Nat Rev Dis Primers. 2018;4:18014. doi:10.1038/nrdp.2018.14

4. Valenzuela PL, Carrera-Bastos P, Galvez BG, et al. Lifestyle interventions for the prevention and treatment of hypertension. Nat Rev Cardiol. 2021;18:251-275. doi:10.1038/s41569-020-00437-9

5. Carey RM, Muntner P, Bosworth HB, Whelton PK. Prevention and control of hypertension: JACC health promotion series. $\mathrm{J} \mathrm{Am} \mathrm{Coll}$ Cardiol. 2018;72:1278-1293. doi:10.1016/j.jacc.2018.07.008

6. Yan B, Peng L, Dong Q, et al. Reverse-dipper pattern of blood pressure may predict lacunar infarction in patients with essential hypertension. Eur J Neurol. 2015;22:1022-1025. doi:10.1111/ ene. 12659

7. Han B, Chen WZ, Li YC, Chen J, Zeng ZQ. Sleep and hypertension. Sleep Breath. 2020;24:351-356. doi:10.1007/s11325-019-01907-2

8. Palagini L, Bruno RM, Gemignani A, Baglioni C, Ghiadoni L, Riemann D. Sleep loss and hypertension: a systematic review. Curr Pharm Des. 2013;19:2409-2419. doi:10.2174/ 1381612811319130009

9. Lo K, Woo B, Wong M, Tam W. Subjective sleep quality, blood pressure, and hypertension: a meta-analysis. J Clin Hypertens. 2018;20:592-605. doi:10.1111/jch.13220

10. Wang L, Hu Y, Wang X, Yang S, Chen W, Zeng Z. The association between sleep duration and hypertension: a meta and study sequential analysis. J Hum Hypertens. 2020;35:621-626. 
11. Li X, Sotres-Alvarez D, Gallo LC, et al. Associations of sleep-disordered breathing and insomnia with incident hypertension and diabetes. The Hispanic community health study/study of latinos. Am J Respir Crit Care Med. 2021;203:356-365. doi:10.1164/ rccm.201912-2330OC

12. Sadeh A. The role and validity of actigraphy in sleep medicine: an update. Sleep Med Rev. 2011;15:259-267. doi:10.1016/j. smrv.2010.10.001

13. Doyle CY, Ruiz JM, Taylor DJ, et al. Associations between objective sleep and ambulatory blood pressure in a community sample. Psychosom Med. 2019;81:545-556. doi:10.1097/ PSY.0000000000000711

14. Quan SF, Howard BV, Iber C, et al. The sleep heart health study: design, rationale, and methods. Sleep. 1997;20:1077-1085.

15. Abad VC, Guilleminault C. Diagnosis and treatment of sleep disorders: a brief review for clinicians. Dialogues Clin Neurosci. 2003;5:371-388.

16. Smurra MV, Dury M, Aubert G, Rodenstein DO, Liistro G. Sleep fragmentation: comparison of two definitions of short arousals during sleep in OSAS patients. Eur Respir J. 2001;17:723-727. doi:10.1183/ 09031936.01.17407230

17. Wang Y, Carreras A, Lee S, et al. Chronic sleep fragmentation promotes obesity in young adult mice. Obesity (Silver Spring). 2014;22:758-762. doi:10.1002/oby.20616

18. Koolhaas CM, Kocevska D, Te Lindert BHW, et al. Objectively measured sleep and body mass index: a prospective bidirectional study in middle-aged and older adults. Sleep Med. 2019;57:43-50. doi:10.1016/j.sleep.2019.01.034

19. Morrell MJ, Finn L, Kim H, Peppard PE, Badr MS, Young T. Sleep fragmentation, awake blood pressure, and sleep-disordered breathing in a population-based study. Am J Respir Crit Care Med. 2000;162:2091-2096. doi:10.1164/ajrccm.162.6.9904008

20. Ramos AR, Weng J, Wallace DM, et al. Sleep patterns and hypertension using actigraphy in the Hispanic community health study/study of latinos. Chest. 2018;153:87-93. doi:10.1016/j.chest.2017.09.028

21. Haas DC, Foster GL, Nieto FJ, et al. Age-dependent associations between sleep-disordered breathing and hypertension: importance of discriminating between systolic/diastolic hypertension and isolated systolic hypertension in the Sleep Heart Health Study. Circulation. 2005;111:614-621. doi:10.1161/01.CIR.0000154540.62381.CF
22. Sanders MH, Newman AB, Haggerty CL, et al. Sleep and sleep-disordered breathing in adults with predominantly mild obstructive airway disease. Am J Respir Crit Care Med. 2003;167:7-14. doi:10.1164/rccm.2203046

23. Cox NS, Pepin V, Holland AE. Greater sleep fragmentation is associated with less physical activity in adults with cystic fibrosis. J Cardiopulm Rehabil Prev. 2019;39:E11-E14. doi:10.1097/ HCR.0000000000000363

24. Hursel R, Rutters F, Gonnissen HK, Martens EA, WesterterpPlantenga MS. Effects of sleep fragmentation in healthy men on energy expenditure, substrate oxidation, physical activity, and exhaustion measured over $48 \mathrm{~h}$ in a respiratory chamber. Am J Clin Nutr. 2011;94:804-808. doi:10.3945/ajcn.111.017632

25. van den Berg JF, Knvistingh Neven A, Tulen JH, et al. Actigraphic sleep duration and fragmentation are related to obesity in the elderly: the Rotterdam study. Int $J$ Obes (Lond). 2008;32:1083-1090. doi:10.1038/ijo.2008.57

26. Yan B, Zhao B, Fan Y, et al. The association between sleep efficiency and diabetes mellitus in community-dwelling individuals with or without sleep-disordered breathing. J Diabetes. 2020;12:215-223. doi:10.1111/1753-0407.12987

27. Castro-Diehl C, Diez Roux AV, Redline S, et al. Sleep duration and quality in relation to autonomic nervous system measures: the multi-ethnic study of atherosclerosis (Mesa). Sleep. 2016;39:1927-1940. doi:10.5665/sleep.6218

28. Ross AJ, Yang H, Larson RA, Carter JR. Sleep efficiency and nocturnal hemodynamic dipping in young, normotensive adults. $\mathrm{Am}$ J Physiol Regul Integr Comp Physiol. 2014;307:R888-92. doi:10.1152/ajpregu.00211.2014

29. Cao Z, Shen L, Wu J, et al. The effects of midday nap duration on the risk of hypertension in a middle-aged and older Chinese population: a preliminary evidence from the Tongji-Dongfeng Cohort Study, China. J Hypertens. 2014;32:1993-8; discussion 1998. doi:10.1097/ НJH.0000000000000291

30. Peacock J, Diaz KM, Viera AJ, Schwartz JE, Shimbo D. Unmasking masked hypertension: prevalence, clinical implications, diagnosis, correlates and future directions. $J$ Hum Hypertens. 2014;28:521-528. doi:10.1038/jhh.2014.9
Nature and Science of Sleep

\section{Publish your work in this journal}

Nature and Science of Sleep is an international, peer-reviewed, open access journal covering all aspects of sleep science and sleep medicine, including the neurophysiology and functions of sleep, the genetics of sleep, sleep and society, biological rhythms, dreaming, sleep disorders and therapy, and strategies to optimize healthy sleep.
The manuscript management system is completely online and includes a very quick and fair peer-review system, which is all easy to use. Visit http://www.dovepress.com/testimonials.php to read real quotes from published authors. 